\title{
Prevalence and antibiotic resistance profile of Shiga-toxigenic Escherichia coli 0157 (STEC) from retailed miscellaneous meat and fish types in Abuja, Nigeria
}

\section{Adaeze Joy Alua, Gabriel K. Omeizaa, James A. Ameh ${ }^{b}$, Enem S.I ${ }^{b}$}

a Department of Veterinary Public Health and Preventive Medicine, Faculty of Veterinary Medicine, University of Abuja, F.C.T., Nigeria.

b Department of Veterinary Microbiology, Faculty of Veterinary Medicine, University of Abuja, F.C.T., Nigeria.

\begin{tabular}{lll}
\hline & & \\
Received: $27 / 1 / 2021$ & Revised: 13/3/2021 & Accepted: 15/4/2021
\end{tabular}

DOI: https://doi.org/10.31559/VMPH2021.2.2.2 
Available online at www.refaad.com

VMPH 2(2); 2021: 37-43

Research Article

Veterinary Medicine and Public Health Journal (VMPH)

Journal Homepage: https://www.refaad.com/views/vmph/home.aspx

www.refaad.com

ISSN : 2707-7195(Online) 2707-7187(Print)

\title{
Prevalence and antibiotic resistance profile of Shiga-toxigenic Escherichia coli 0157 (STEC) from retailed miscellaneous meat and fish types in Abuja, Nigeria
}

\author{
Adaeze Joy Alu*a, Gabriel K. Omeizaa, James A. Ameh', Enem S.I ${ }^{\text {b }}$ \\ a Department of Veterinary Public Health and Preventive Medicine, Faculty of Veterinary Medicine, University of Abuja, \\ F.C.T., Nigeria. \\ ${ }^{b}$ Department of Veterinary Microbiology, Faculty of Veterinary Medicine, University of Abuja, F.C.T., Nigeria. \\ Emails: ${ }^{a}$ adaeze.alu@uniabuja.edu.ng, ${ }^{b}$ gabriel.omeiza@uniabuja.edu.ng, ${ }^{c}$ jamesameh10@gmail.com
}

* Corresponding author: Alu, AJ., E-mail: adaeze.alu@uniabuja.edu.ng

How to cite this article: Alu, AJ. et al., Prevalence and antibiotic resistance profile of Shiga-toxigenic Escherichia coli O157 (STEC) from retailed miscellaneous meat and fish types in Abuja, Nigeria. Veterinary Medicine and Public Health Journal 2(2); 2021: 37-43.

DOI: https://doi.org/10.31559/vmph2021.2.2.2 Received Date: 27/1/2021 Revised Date: 13/3/2021 Accepted Date: 15/4/2021

\section{Abstract}

Most Escherichia coli strains are harmless intestinal bacteria of animals, but some are implicated in food infection/poisoning especially Shiga toxin (or Vero toxin) producing E. coli (STEC) due to consumption of meat. This study was conducted to determine the prevalence and antibiotic resistance profile of Shigatoxigenic Escherichia coli 0157 (STEC) from retailed miscellaneous fish and meat types in Abuja, Federal Capital Territory, Nigeria. A total of 256 meat and fish consisting of cow muscles, intestines, rumen-sacs, livers and tails, cat-fish, frozen fish (mackerel and herrings) were examined. Escherichia coli were isolated by enrichment culture cefixime-tellurite sorbitol MacConkey agar (CT-SMAC), morphological, biochemical, serotype latex agglutination and disk diffusion methods. Of the 256 samples, 138 (53.9\%) were contaminated with E. coli and $28(21.7 \%)$ E. coli strains were positive for Shigatoxigenic Escherichia coli 0157 (STEC). Meat muscles had the highest prevalence of STEC (7.41\%) among meat samples, followed by rumen-sacs (6.0\%), intestines (5.77\%), tails (4.0\%), and the prevalence of STEC in Fish includes Cat-fish intestine (26.7\%), skin (21.4\%), Mackerel intestine (26.7\%), skin (14.3\%), and Herrings skin (15.4\%), gill (7.1\%). All the STEC assessed indicated multi-drug resistance, with the isolates showing $100 \%$ resistant to ampicilin, and erythromycin, nitrofurantoin (95.7\%), amoxicilin clavulanic acid (84.3\%), sulphamethaxazole/trimethoprim (75\%), streptomycin (75\%), tetracycline (66.17\%), and gentamycin (53.6\%). The isolates were susceptible to ciprofloxacin (66.7\%), Cefoxitin (66.7\%), amikacin (39.3\%), and chloramphenicol (35.7\%). The implication of STEC in this study suggests that contaminated meat types are sold to consumers and can result to serious foodborne hazards. Prescription of ciprofloxacin and cefoxicilin are recommended against this organism. Application of good hygienic procedures in meat and fish handling processes and proper boiling before consumption can mitigate the risk of infection due to resistance STEC strains.

Keywords: Serotyping; Latex agglutination; Antibiotic-resistance; Escherichia coli. 


\section{Introduction}

Escherichia coli a facultative anaerobe usually found in the gastrointestinal tract of mammals, belonging to the family Enterobacteriaceae, (CDC, 2012). The emergence of 0157 strains of Escherichia coli poses serious threat to public health with regards to their devastating and zoonotic importance, (Wasteson, 2001; Alexis et al. 2010). Escherichia coli 0157:H7 is a highly virulent Enterohemorrhagic Escherichia coli with an acronym (EHEC) used to denote a sub-set of Shiga toxin (Stx)-producing E. coli (STEC) also recognized as vero toxin producing E. coli (VTEC), which causes serious disease conditions in humans including hemorrhagic colitis, bloody diarrhea and the hemolytic uremic syndrome (HUS), (Wasteson, 2001). E. coli 0157:H7 was first isolated from hamburger and cattle as reported by the Centers for Disease Control (CDC 1982) which are considered to be the prominent reservoir for EHEC 0157. E. coli 0157 is commonly found in the intestines of ruminants and cross contamination of any parts of the animal is possible when meat processing is not properly done, ruminants are usually exposed to STEC due to contaminated feed and drinking water as well as exposure to the feces of other animals shedding the bacteria (LeJeune et al. 2001; Persad and LeJeune 2014). The bacterium (E. coli 0157) is also found naturally in the intestines of other animals like pigs, sheep, goats and deer as well as in milk, vegetables and fish (Govindarajan, 1990; WHO, 2004; Thampuran et al. 2005; Yousuf, 2008; Shafiullah et al. 2018; Yakubu, 2018). Few studies have evaluated antimicrobial resistance of $E$. coli 0157 in Sub-Saharan Africa. Shigatoxin-producing $E$. coli 0157 has become a major meat safety issue worldwide, (Nakazawa et al. 1999; Okeke et al. 2000).

Meat and fish are major staple in Nigerian food, according to government estimates, Nigeria, consumes beef in tone of over 360,000 tunes each year, accounting for half of all West Africa, with emerging middle-class population of over 200 million people. There is a booming demand for meat and fish in Nigeria, consumption of meat and fish is low compared with advanced economies in percapita terms, but growing fast in alarming rate and expected to quadruple by 2050, (FAO, 2017). Unwholesome meat can constitute great degree of health hazard to its consumer, especially when implicated with Shiga-toxin producing $E$. coli strains, (WHO, 2018). There is dart of information on these virulent and resistance strain of $E$. coli from meat and fish in Abuja. This paper, therefore, reports on the prevalence and antibiotic profile of Shigatoxigenic Escherichia coli 0157 (STEC) from meat and fish in Abuja, Nigeria, to highlight the potential threat to public health and safety.

\section{Materials and Methods}

\section{Area/Sources and collection of samples:}

This study was carried out in Abuja, Federal Capital Territory (FCT), which is made up of six Area councils. Geographically, F.C.T. is placed at the latitude 9.0578499 and longitude 7.49508, in the northern hemisphere. Sharing borders with Niger state to the west/north, Kaduna state to the north/east, Nasarawa state to the east/south and Kogi state to the south/west, covering a landmass of approximately $7,315 \mathrm{~km}^{2}$, with moderate climatic conditions, having estimated population of $1,406,239$ as at the 2006 population census (NPC, 2006). The total of 256 meat $(n=128)$ and fish $(n=128)$ samples was used for this study, collected from local markets in three area councils of F.C.T. randomly selected, namely; Kwali, Gwagwalada, and Bwari Area Councils. All meat and fish samples were bought in wraps as they would normally be sold to the consumer from the various markets and were appropriately labeled, placed in a flask with ice, then transported immediately for analysis in the laboratory between 2 to 6 hours after collection.

\section{Bacteriological examination:}

About $10 \mathrm{~g}$ of samples each were stomached and diluted in $90 \mathrm{ml}$ of peptone water (Merck), then cultured overnight at $37^{\circ} \mathrm{C}$. Prepared sample broth culture was plated onto MacConkey agar, Eosin methylene blue agar (EMB) (Oxoid) for the identification of the green metallic sheen morphological characteristics of $E$. coli colonies. The colonies were further inoculated into Sorbitol MacConkey agar (Oxoid) enriched with cefixime tellurite supplement (CT-SMAC) (Oxoid) to selectively distinguish the non-sorbitol fermenting $E$. coli 0157 strains from other $E$. coli strains isolated, each sample was streaked onto the media surface and incubated at $37^{\circ} \mathrm{C}$ for 24 hours (Janet et al. 2003). The morphological characteristics, sorbitol fermentation, gram staining and motility of the colonies were tested. Biochemical tests on the presumptive E. coli colonies were performed and the isolates were identified according to standard methods, (Cheesbrough, 2006), then stored in the refrigerator at $4^{\circ} \mathrm{C}$ on slants of nutrient agar for further work.

\section{Serotyping of the Isolates:}

Characterization of the STEC serotype was done by slide agglutination with antisera according to the method of Nataro and Kaper (1998). The shigatoxigenic E. coli 0157 antisera rapid latex agglutination test kit (Oxoid) was used to serotype E. coli strains. With drops of antisera on slide trays with wells, colonies were examined for the shigatoxigenic E. coli 0157 . The isolates were tested using the control latex reagents for nonspecific agglutination of organisms with latex. Positively reactive 0157 colonies were transferred to other 
slant medium for further testing, which allows for more yield of the bacterial growth on which to perform more 0157 agglutination assay. Strains that agglutinate with latex reagents were considered as E. coli 0157 serotype, (Blanco et al. 2003).

\section{Antibiotic Resistance testing:}

Antimicrobial susceptibility testing of the $E$. coli 0157 isolates to different antimicrobial agents was performed according to the Clinical and laboratory standards institute guidelines and the agar disk diffusion method (CLSI, 2015; Bauer et al. 1966), with 12 commercially available antimicrobial agents (Oxiod) on Mueller-hinton agar (USA), which includes ciprofloxacin (CIP) $5 \mu \mathrm{g}$, erythromycin (E) $15 \mu \mathrm{g}$, ampicillin (AMP) $10 \mu \mathrm{g}$, amoxicillin/calvulanic acid (AMC) $30 \mu \mathrm{g}$, sulphamethoxazole/trimethoprim (SXT) $25 \mu \mathrm{g}$, cefoxitin (FOX) $30 \mu \mathrm{g}$, tetracycline (TE) $30 \mu \mathrm{g}$, amikacin (AK) $30 \mu \mathrm{g}$, streptomycin (S) $10 \mu \mathrm{g}$, nitrofurantoin (F) $300 \mu \mathrm{g}$, chloramphenicol (C) 30 $\mu \mathrm{g}$, and gentamicin (CN) $10 \mu \mathrm{g}$. The agar plates were prepared according to the manufacturer guidelines, with a sterile glass spreader, broth culture was spread gently over the surface of agar plates and allowed to dry for $5 \mathrm{~min}$. The antibiotic discs were firmly placed on the agar surface with $1 \mathrm{~cm}$ distance apart and incubated at $37^{\circ} \mathrm{C}$ for 20 hours. The diameter of inhibition zone formed around each disc was measured and evaluated according to CLSI (2015).

\section{Statistical Analysis:}

Chi square analysis (in Statistical package for social sciences; version 20.0) was used to test associations in means of different retailed meat and fish types with locations where they were sampled at $95 \% \mathrm{CI}$, such that values less than $0.05(\mathrm{P}<0.05)$ was considered significant.

\section{Results}

Detection of $E$. coli: Table 1; shows the prevalence of $E$. coli in various types of meat and fish studied. From the results, $58.59 \%(n=75 / 128)$ meat and $49.21 \% \quad(n=63 / 128)$ fish samples harbored $E$. coli strains, totaling $53.9 \%(n=138 / 256)$ meat and fish samples. There were statistically significant differences observed in the frequency of $E$. coli in various samples $(\mathrm{P}<0.05)$.

Detection of STEC: Table 2 and 3; characterizes the prevalence of shigatoxigenic E. coli 0157 in meat and fish samples with overall prevalence of $10.9 \%$ $(n=28 / 256)$, out of $138 \mathrm{E}$. coli isolates $20.3 \%$ $(\mathrm{n}=28 / 138)$ strains were characterized and confirmed as $E$. coli 0157 serotypes, with the prevalence of $9.4 \%(n=12 / 128)$ in meat and $12.5 \%$ $(n=16 / 128)$ from fish. Frequency of E. coli 0157 cluster in various Meat types includes cow meat muscles (11.5\%), intestine (15.4\%), rumen sac (11.5\%), tail muscles (4.0\%), and liver (0\%), while, 0157 serotype in Fish types includes Cat-fish intestine (26.6\%), skin (21.4\%), gill $(0 \%)$, and muscles (0\%), Mackerel intestine $(14.2 \%)$, skin $(14.2 \%)$, gill $(14.2 \%)$, and muscles $(0 \%)$, and Herrings skin (14.2\%), gill (7.14\%), intestine (0\%), and muscles (0\%). E. coli 0157 was implicated in some meat and fish studied with statistically significant differences observed $(\mathrm{P}<0.05)$.

Table (1): Meat and fish types showing prevalence of $E$. coli

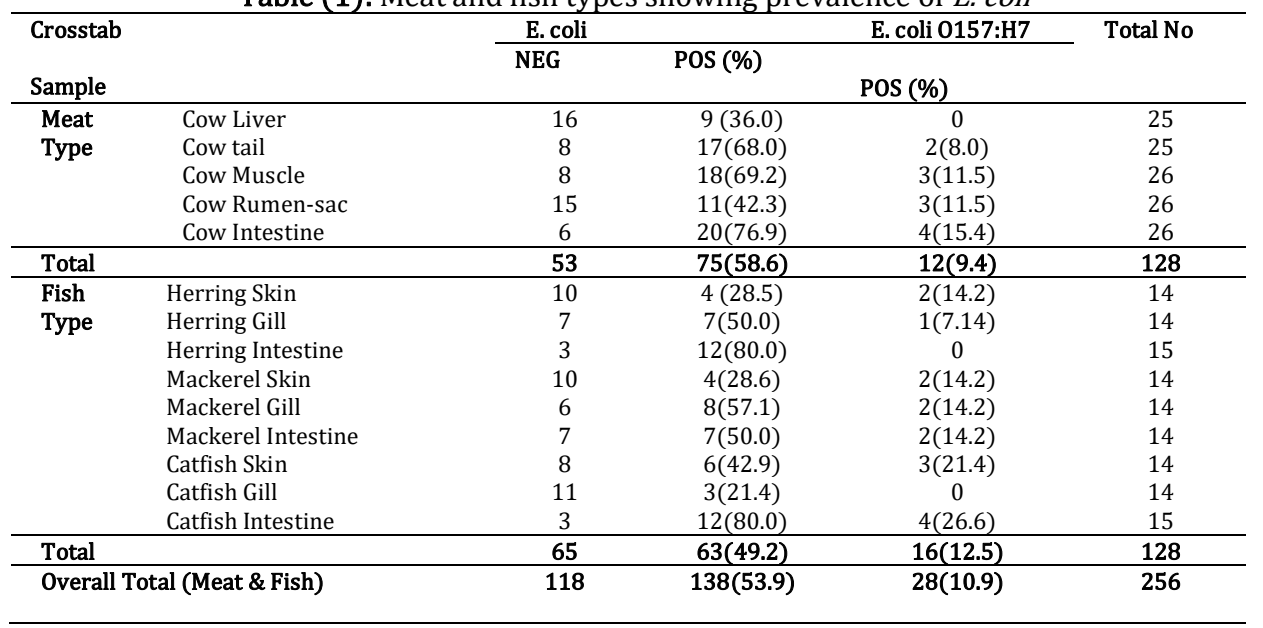


Table (2): Sample Meat \& Fish * E. coli 0157:H7 Cross tabulation

\begin{tabular}{|c|c|c|c|c|c|}
\hline \multirow{4}{*}{ Sample } & \multirow[b]{3}{*}{ Meat } & \multirow{4}{*}{$\begin{array}{l}\text { Count } \\
\text { Expected Count }\end{array}$} & \multicolumn{2}{|c|}{ E. coli 0157:H7 } & \multirow[b]{2}{*}{ Total } \\
\hline & & & NEG & POS & \\
\hline & & & 116 & 12 & 128 \\
\hline & & & 114.0 & 14.0 & 128.0 \\
\hline \multirow{4}{*}{ Total } & Fish & Count & 112 & 16 & 128 \\
\hline & & Expected Count & 114.0 & 14.0 & 128.0 \\
\hline & & Count & 228 & 28 & 256 \\
\hline & & Expected Count & 228.0 & 28.0 & 256.0 \\
\hline
\end{tabular}

\begin{tabular}{lllc} 
Chi-Square Tests & Value & $\mathrm{df}$ & Asymptotic Significance (2-sided) \\
\cline { 2 - 3 } Pearson Chi-Square & $.642^{\mathrm{a}}$ & 1 & .423 \\
Continuity Correction & .361 & 1 & .548 \\
Likelihood Ratio & .644 & 1 & .422 \\
Fisher's Exact Test & & & .424 \\
Linear-by-Linear Association & .639 & 1 & \\
N of Valid Cases & 256 & & \\
\hline
\end{tabular}

contamination due to E. coli 0157 in fish is not

The calculated value of Chi-square is 0.642 and statistically different from that of beef.

the $\mathrm{P}$-value is 0.423 which is greater than the level of significance of $0.05(5 \%)$, we accept the null $\mathrm{H}_{0} 1$ : The level of contamination due to E. coli 0157 hypothesis and conclude that the level of

in fish is not statistically different from that of beef.

Table (3): $\mathrm{H}_{0} 2$ : No statistically significant different observed in the level of contamination due to E. coli 0157 among the different types of fish used for the study.

\begin{tabular}{|c|c|c|c|c|c|c|}
\hline & & & & \multicolumn{2}{|c|}{ E. coli 0157} & \multirow[b]{2}{*}{ Total } \\
\hline & & & & NEG & POS & \\
\hline \multirow{18}{*}{ Fish Type } & Herring Skin & Count & & 12 & 2 & 14 \\
\hline & & Expected Count & & 12.3 & 1.8 & 14.0 \\
\hline & Herring Gill & Count & & 13 & 1 & 14 \\
\hline & & Expected Count & & 12.3 & 1.8 & 14.0 \\
\hline & Herring Intestine & Count & & 15 & 0 & 15 \\
\hline & & Expected Count & & 13.1 & 1.9 & 15.0 \\
\hline & Mackerel Skin & Count & & 12 & 2 & 14 \\
\hline & & Expected Count & & 12.3 & 1.8 & 14.0 \\
\hline & Mackerel Gill & Count & & 12 & 2 & 14 \\
\hline & & Expected Count & & 12.3 & 1.8 & 14.0 \\
\hline & Mackerel Intestine & Count & & 12 & 2 & 14 \\
\hline & & Expected Count & & 12.3 & 1.8 & 14.0 \\
\hline & Catfish Skin & Count & & 11 & 3 & 14 \\
\hline & & Expected Count & & 12.3 & 1.8 & 14.0 \\
\hline & Catfish Gill & Count & & 14 & 0 & 14 \\
\hline & & Expected Count & & 12.3 & 1.8 & 14.0 \\
\hline & Catfish Intestine & Count & & 11 & 4 & 15 \\
\hline & & Expected Count & & 13.1 & 1.9 & 15.0 \\
\hline \multirow{2}{*}{\multicolumn{2}{|c|}{ Total }} & Count & & 112 & 16 & 128 \\
\hline & & Expected Count & & 112.0 & 16.0 & 128.0 \\
\hline Chi-Square Tes & & Value & Df & \multicolumn{3}{|c|}{ Asymptotic Significance (2-sided) } \\
\hline $\begin{array}{l}\text { Cni-square lests } \\
\text { Pearson Chi-Square }\end{array}$ & & $8.446^{\mathrm{a}}$ & 8 & \multicolumn{3}{|c|}{.391} \\
\hline \multicolumn{2}{|c|}{ Likelihood Ratio } & 11.370 & 8 & \multicolumn{2}{|l|}{.182} & \\
\hline \multicolumn{2}{|c|}{ Linear-by-Linear Association } & 1.219 & 1 & \multicolumn{2}{|l|}{.270} & \\
\hline \multicolumn{2}{|c|}{$\mathrm{N}$ of Valid Cases } & 128 & & & & \\
\hline
\end{tabular}

The calculated value of Chi-square is 8.446 and the P-value is 0.391 being greater than the level of significance of $0.05(5 \%)$, we therefore accept the null hypothesis and conclude that there is no statistically significant difference in the levels of contamination due to E. coli 0157 among the different type of fish used for the study.

Antibiotic Sensitivity: Figure 1; highlights the multidrug resistance pattern of E. coli 0157 in this study. The $E$. coli isolates exhibited $100 \%$ resistant to ampicilin, and erythromycin, nitrofurantoin (95.7\%), amoxicilin clavulanic acid (84.3\%), sulphamethaxazole/trimethoprim (75\%), streptomycin (75\%), tetracycline $(66.17 \%)$, and gentamycin (53.6\%). The isolates were susceptible to ciprofloxacin (66.7\%), cefoxitin (66.7\%), amikacin (39.3\%), and chloramphenicol (35.7\%). See Figure 1. 


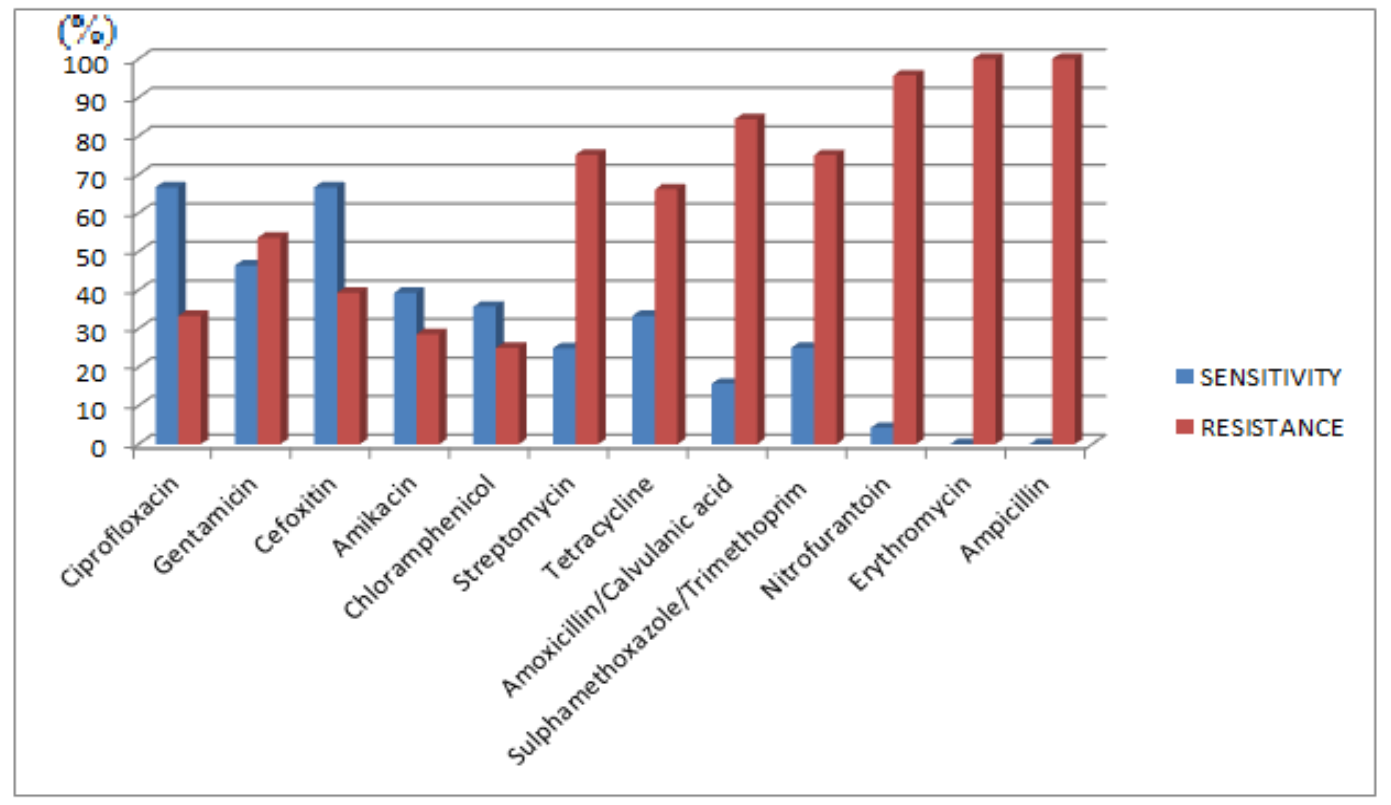

Figure (1): Percentage distribution of resistance pattern of E. coli 0157 isolates to tested antibiotics

\section{Discussion}

The prevalence of STEC 0157:H7 in animal and food products from almost all over Africa poses great risk for human infection (Athumani, 2017); the current surveillance system worldwide reveals the diversity and impact of STEC infections as well as sources of contamination. High increase in STEC outbreaks over the past 10 years due to contaminated food and contact with animals and animal products is a public health concern, (JunSeob, 2020). The need to understand animals and food sources as potential reservoir for STEC is quite essential.

Of the 256 meat and fish studied, results show prevalence rate of $10.9 \%$ (28) STEC strains, among these number $12(9.4 \%)$ were from meat and 16 $(12.5 \%)$ from fish. This is in agreement with similar work done by Kumar (2004) on seafood and beef in Mangalore. Among these number 12 (9.4\%) were from meat and 16 (12.5\%) from fish. Also Ameh et al. (2002), isolated shigatoxigenic E. coli 0157 from both diarrheic infants and calves from Maiduguri, at the prevalence of $10.53 \%$, where they reported that the prevalence declined with increase in age.

In this study, the prevalence of STEC in meat agrees with 10\% STEC prevalence from beef and rectal swabs reported by Shafiullah et al. (2018) in Bangladesh, but higher than the $3.5 \%$ prevalence in ground beef reported by Alzira et al. (2007), however contrary to this work, Mashak (2018) reported higher prevalence of $14 \%$ in raw meat.

We recorded $12.5 \%$ prevalence of STEC in various types of fresh fish and frozen fish from retail markets, these is contrary to similar work done by Thampuran et al. (2013), who worked on 23 different types of fresh and frozen fish from retail markets, reporting E. coli 0157 strain to be absent but had the presence of other MUG and sorbitalnegative and virulent types of $E$. coli suggesting for further studies in fish. Also, Kumar et al. (2010) screened E.coli isolated from fish, clams, and water for specific genes of stx, hlyA and rfb0157; $\mathrm{He}$ reported that $5 \%$ clam and $3 \%$ fresh fish samples were positive for non-0157 STEC. However, this study agrees with the work done by Gupta et al. (2013), who isolated STEC from raw fish and fish products from retail markets of the Ludhiana, purporting raw fish to be the major source for virulence gene of STEC. Similarly, Surendraraj et al. (2010) reported significant incidence of shigatoxigenic Escherichia coli in fish and shrimp from different retail fish markets in Cochin. Generally there is paucity of literature on STEC in fish in this region.

The antimicrobial resistance pattern in this study revealed that there is multiple drug resistance of the isolates to most of the antibiotics used, but was mostly susceptible to ciprofloxacin and cefoxitin. Globally, antimicrobial resistances are now observed in high frequencies, (Mukherjee et al. 2017), several studies implicated stx1 gene as encoding antimicrobial (AMR) in STEC 0157.

\section{Conclusion}

This study provides evidence of contamination of common street vented edible meat and fish types circulating in local markets of the Area councils constituting the Federal Capital Territory. Documented prevalence of $E$. coli varied amongst the different sampled area councils.

This study provides evidence that toxigenic Escherichia coli 0157 strains are also common contaminants of meat and fish types in selected Area councils of Abuja. The antibiotic resistance strain of E. coli 0157 found in this study are of public health importance, therefore, there is urgent need to 
legislate to assure compliance on hygiene on street vended meat and fish types in the FCT.

There is need for public awareness on the epidemiology of the pathogen. Appropriate hygienic measures must be imbibed by handlers of meat and fish products. Proper cooking of meat and fish before consumption is highly recommended.

This study suggests a wide collaborative study of all street retailed meat and fish products to understand sources of food contaminants and policies to mitigate them.

Acknowledgements: We acknowledge Dr. Mohammed Balarabe for his immense contributions to this work. The staff of Biotechnology advance laboratory, Sheda science and technology complex (SHESTCO), Sheda, Abuja, for enabling good research environment and atmosphere.

Conflicts of Interests: There are no conflicts of interest between the authors regarding the publication of this paper.

\section{Reference}

1. Alexis Gaicia, James G. Fox, Thomas E. Besser, (2010). Zoonotic Enterohemorrhagic Escherichia coli: A One Health Perspective, ILAR Journal, 51(3):221-232, https://doi.org/10.1093/ilar.51.3.221.

2. Alzira Maria Morato Bergamini; Marise Simões; Kinue Irino; Tânia Aparecida Tardelli Gomes \& Beatriz Ernestina Cabilio Guth (2007). Prevalence and characteristics of Shiga toxin-producing Escherichia coli (STEC) strains in ground beef in São Paulo, Brazil. Braz.J. Microbiol. 38(3). doi.org/10.1590/S1517-83822007000300032.

3. Ameh J. A., Zaria L. T. \& Mamman I., (2002). Prevalence of Shiga toxigenic Escherichia coli (STEC) 0157 in Diarrhoeic Infants and Calves in Maiduguri, Nigeria. Nigerian Journal Experimental and Applied Biology, 3 (1): 23-27.

4. Athumani M Lupindu (2017). Epidemiology of Shiga toxin-producing Escherichia coli 0157:H7 in Africa in review. J. Southern African Journal of Infectious Diseases. Doi10.1080/23120053.2017.1376558.

5. Bauer A.W, Kirby W.M.M, Sheris J.C \& Truck M. (1966). Antibiotic susceptibility testing by a standardized single disc method. American Journal of Clinical Pathology. 45:493-496. https://doi.org/10.1093/ajcp/45.4_ts.493.

6. Blanco M., Blanco J. E., Mora A., Rey J., Alonso J. M. \& Hermoso M. (2003). Serotypes, virulence genes, and intimin types of Shiga toxin (verotoxin)-producing Escherichia coli isolates from healthy sheep in Spain. J. Clin. Microbiol. 41 1351-1356. 10.1128/JCM.41.4.1351-1356.2003.

7. Centers for Disease Control and prevention (1982). Epidemiologic Notes and Reports: Isolation of Escherichia coli 0157: H7 from Sporadic Cases of Hemorrhagic Colitis-United States. Morbidity and Mortality Weekly Report 31:580-585.

8. Centers for Disease Control and prevention (2012). National Center for Emerging and Zoonotic Infectious Diseases. Retrieved 2 October.
9. Cheesbrough, M. (2006). District Laboratory Practice in Tropical Countries (2nd Edition). London English Language Book Society. pp. 100-194.

10. CLSI (2015). Clinical and Laboratory standards Institute; Wayne, PA: p. S100-S125.

11. Food and Agriculture Organization (FAO), (2017). FAO Global Perspectives studies Unit, forthcoming. Africa Sustainable Livestock (ASL) 2050 Country Brief NIGERIA, Food and Agriculture Organization of the United Nations, Rome.

12. Govindarajan C. V (1990). Maintenance of hygienic and sanitary conditions including personal hygiene in the meat factory. Technical paper in First National Seminar on Marketing of Meat Food Products in India, Aligarh, India

13. Gupta B., Ghatak S. \& Gill J.P.S (2013). Incidence and virulence properties of $E$. coli isolated from fresh fish and ready-to-eat fish products, Vet World 6(1):5-9. doi:10.5455/vetworld.2013.5-9.

14. Janet E.L. Corry, G.D.W. Curtis \& Rosamund M. Baird, (2003). Cefixime tellurite sorbitol MacConkey (CTSMAC) agar, Progress in Industrial Microbiology, Elsevier, 37: 422-424, doi/10.1016/S0079-6352.

15. Jun-Seob K., Moo-Seug L. \& JiHyung K. (2020). Recent Updates on Outbreaks of Shiga Toxin-Producing Escherichia coli and Its Potential Reservoirs. Front. Cell. Infect. Microbiol., 04. doi.org/10.3389/fcimb.2020.00273.

16. Kumar H.S. (2004). Characterization of Shiga toxinproducing Escherichia coli (STEC) isolated from seafood and beef. FEMS J. Microbilogy Letters, 23(1).

17. Kumar, H., Sanath, Otta, S.K, Karunasagar, I. \& Karunasagar. (2009). Detection of Shiga-toxigenic Escherichia coli (STEC) in fresh seafood and meat marketed in Mangalore, India by PCR. Letters in Applied Microbiology, 33(5): 334-38. https://doi.org/10.1046/j.1472-765x.2001.01007.x.

18. LeJeune, J. T., Besser, T. E., Merrill, N. L., Rice, D. H. \& Hancock, D. D. (2001). Livestock drinking water microbiology and the factors influencing the quality of drinking water offered to cattle. J. Dairy Sci. 84, 1856-1862. doi: 10.3168/jds.S0022-0302(01) 74626-7.

19. Mashak Z. (2018). Virulence Genes and Phenotypic Evaluation of the Antibiotic Resistance of Vero Toxin Producing Escherichia coli Recovered from Milk, Meat, and Vegetables, Jundishapur J Microbiol.11(5): e62288. doi: 10.5812/jjm.62288.

20. Md. Shafiullah Parvej, Montasir Mamun, Jayedul Hassan, Md. Muket Mahmud, Marzia Rahman, Md. Tanvir Rahman, Md. Bahanur Rahman \& K. H. M. Nazmul Hussain Nazir, (2018). Prevalence and characteristics of Shiga-toxin producing Escherichia coli (STEC) isolated from beef slaughterhouse. Journal of Advanced Veterinary and Animal Research 5(2): 218-225.4, DOI: https://doi.org/10.5455/javar.2018.e27.

21. Mukherjee S, Mosci, RE, Anderson CM, Snyder BA, Collins J, Rudrik JT \& Manning SD (2017). Antimicrobial Drug-Resistant Shiga-Toxin-Producing Escherichia coli Infections in Michigan, United States of America. Emerging Infectious Disease 23 (9): 1609-1611. 
22. Nakazawa M., Akiba M. \& Sameshima T., (1999). Swine as a potential reservoir of shigatoxinproducing Escherichia coli 0157:H7 in Japan. Emerging Infectious Disease, 5 (6): 833-845.

23. Nataro, J.P. \& Kaper, J.B. (1998). Diarrheagenic Escherichia coli. Clinical Microbiology Reviews, 11, 142-201.

24. National Population Commission (NPC) 'Enumerators manual', March, (2006).

25. Okeke I.N., Fayinoka S.T. \& Lamikanra A., (2000). Antibiotic resistance in Escherichia coli from Nigerian students, 1986-1998. Emerging infectious Disease, 6(4): 393-395, https://doi.org/10.3201/eid0604.009913.

26. Persad, A. K., \& LeJeune, J. T., (2014). Animal reservoirs of Shiga toxin-producing Escherichia coli. Microbiol. Spectr. 2: EHEC-0027-2014. doi:10.1128/microbiolspec. EHEC-0027-2014.

27. Shafiullah Parvej, Montasir Mamun, Jayedul Hassan, Muket Mahmud, Marzia Rahman, Tanvir Rahman, Bahanur Rahman, K. H. M. \& Nazmul Hussain Nazir, (2018). Prevalence and characteristics of Shiga-toxin producing Escherichia coli (STEC) isolated from beef slaughterhouse. Journal of Advanced Veterinary and Animal Research, 5(2): 218-225. DOI: 10.5455/javar.2018.e271.

28. Surendraraj A. T., Joseph N. \& Toms C., (2010). Molecular Screening, Isolation, and Characterization of Entero-hemorrhagic Escherichia coli 0157:H7 from Retail Shrimp. Journal of food protection.
73(1): 97-103, https://doi.org/10.4315/0362-028x73.1.97.

29. Thampuran N., Surendraraj A. \& Surendran P.K., (2005). Prevalence and characterization of typical and atypical Escherichia coli from fish sold at retail in Cochin, India. J Food Prot. 68(10): 2208-2211, https://doi.org/10.4315/0362-028x-68.10.2208.

30. Wasteson Y. (2001). Zoonotic Escherichia coli. Acta Vet Scand Suppl. 43(1): 79-84, https://doi.org/10.1186/1751-0147-43-s1-s79.

31. World Health Organization (WHO), (2004). Regional Office for Africa Developing and Maintaining Food Safety Control Systems for Africa Current Status and Prospects for Change", Second FAO/WHO Global Forum of food Safety Regulators, Bangkok, Thailand. Pp 12-14.

32. World Health Organization (WHO), (2018). E. coli. World Health Organization E. coli review. 7 February, 2018.

33. Yakubu Y., Shuaibu A.B., Ibrahim A.M., Hassan L.U. \& Nwachukwu R. J., (2018). Risk of Shiga Toxigenic Escherichia coli 0157:H7 Infection from Raw and Fermented Milk in Sokoto Metropolis, Nigeria. Journal of Pathogens. 10: 1155-1160.

34. Yousuf A.H.M., Ahmed M.K., Yeasmi S., Ahsan N., Rahman M.M. \& Islam M.M., (2008). Prevalence of Microbial Load in Shrimp, Penaeus monodon and Prawn, Macrobrachium rosenbergii from Bangladesh. World Journal of Agricultural Sciences. 4 (5): 852-855. 